Correspondence

Nuri Kiraz

nkiraz@ogu.edu.tr
Received 10 June 2009

Accepted 1 May 2010

\section{Evaluation of the GenoType Mycobacteria Direct assay for direct detection of the Mycobacterium tuberculosis complex obtained from sputum samples}

\author{
Nuri Kiraz, Imran Saglik, Abdurrahman Kiremitci, Nilgun Kasifoglu \\ and Yurdanur Akgun
}

Eskisehir Osmangazi University, Faculty of Medicine, Department of Microbiology, Eskisehir, Turkey

\begin{abstract}
An increase in the prevalence of tuberculosis (TB) in recent years has accelerated the search for novel tools for the rapid diagnosis of TB infection. This study evaluated the GenoType Mycobacteria Direct (GTMD) assay (Hain Lifescience) for direct detection of the Mycobacterium tuberculosis complex (MTBC) from sputum samples and compared it with conventional methods. The GTMD test is a commercial assay produced using strip techniques and works based on a nucleic acid sequence-based amplification technique. This test allows $23 S$ rRNA amplificationbased detection of MTBC, Mycobacterium avium, Mycobacterium intracellulare, Mycobacterium kansasii and Mycobacterium malmoense directly from decontaminated clinical samples within $6 \mathrm{~h}$. In the present study, 115 sputum samples were processed to detect acid-fast bacilli (AFB) using two microscopy methods (carbol fuchsin and fluorescent staining), two culture methods [Löwenstein-Jensen (LJ) and BACTEC 12B media] and the GTMD test. The results showed that 86 of the samples were positive by direct microscopy, 84 were positive by BACTEC 12B culture, 73 were positive by $L J$ culture and 95 were positive by the GTMD test. All of the isolates turned out to be MTBC. Moreover, the sensitivity and specificity of the GTMD test for MTBC in patients were 97 and $58 \%$, respectively, taking the culture combination as the gold standard. When the test was compared with culture of samples from anti-TB-treated patients, the sensitivity and specificity for the test were 100 and $15 \%$, respectively. Low specificity in treated people might arise from depressed proliferation of AFB. As the two methods target the same living bacilli, the difference is obviously notable. When the culture results and clinical findings of the patients were evaluated together (true-positive specimens), the sensitivity and specificity values of the GTMD test for all patients were 97 and $90 \%$, respectively. However, both of these values increased to $100 \%$ for the patients receiving anti-TB treatment. These results implied that, to determine whether the patient's sputum contains living AFB, more sensitive techniques should be employed during the follow-up of the patients. These observations suggest that the GTMD method can be useful for early diagnosis of clinically and radiologically suspicious TB cases where smears are negative for Mycobacterium. In addition, the use of a GTMD test in smear-positive cases is helpful and practical in order to identify MTBC quickly. This allows more rapid treatment decisions and infection control precautions.
\end{abstract}

\section{INTRODUCTION}

Tuberculosis (TB) remains an important community health problem. The World Health Organization estimates that 8 million new TB cases are reported annually and are the cause of death in 2-3 million patients. Each untreated pulmonary TB patient is responsible for the spread of the

Abbreviations: AFB, acid-fast bacilli; Gl, growth index; GTMD, GenoType Mycobacteria Direct; NPV, negative predictive value; PPV, positive predictive value; TB, tuberculosis. disease to 10-15 humans over a year. This makes TB one of the most important causes of death from an infectious agent (Corbett et al., 2003; WHO, 2007).

The most effective means of protection is early diagnosis and treatment of the disease. Preliminary diagnosis is based on clinical findings, but definite diagnosis is by laboratory methods (Drobniewski et al., 2003).

The gold standard test in the diagnosis of TB is culture, but biochemical tests used in identification of the species are 
time-consuming. Therefore, molecular methods can be useful in identifying this agent. Over the last decade, several molecular methods have been developed for direct detection and identification of the Mycobacterium tuberculosis complex (MTBC) in clinical specimens (Piersimoni \& Scarparo, 2003).

The GenoType Mycobacteria Direct (GTMD; Hain Lifescience) test is a commercial assay using DNA strip technology and a reverse hybridization method for a line probe assay. This test is used for the molecular identification of mycobacteria directly from decontaminated clinical samples (Franco-Alvarez de Luna et al., 2006). In our study, we aimed to detect the presence of the bacterium earlier by direct microscopic examination using staining with two different methods (carbol fuchsin and a fluorochrome), and with a molecular method, the GTMD test, in sputum samples of infected patients, and to compare this test with conventional culture methods.

\section{METHODS}

Patients and strains. In the present study, we analysed 115 sputum samples obtained from adult patients (28 female and 87 male, human immunodeficiency virus-negative, one sputum sample per patient) with suspicious pulmonary TB (chest radiograph, tuberculin skin test result, clinical presentation) or from TB patients under anti-TB therapy. Sputum specimens were obtained from various clinics of the Medical School of Eskisehir Osmangazi University, Eskisehir, Turkey, and the Taksim/Istanbul Tuberculosis Dispensaries between January 2007 and April 2007. We ensured that the volumes of the sputum samples were sufficient to perform all the diagnostic tests. The samples were stored at $-70{ }^{\circ} \mathrm{C}$ for the GTMD test. All samples were inspected for the presence of acid-fast bacilli (AFB) as determined by direct microscopy and staining with the carbol fuchsin and fluorochrome methods, and were tested for MTBC with the GTMD test (version 4; Hain Lifescience). All samples were also inoculated onto LöwensteinJensen (LJ) solid medium (Dimed) and into BACTEC 12B liquid cultures (BACTEC 460 TB; Becton Dickinson Diagnostic Instruments).

This study was approved by the Ethics Committee of Eskisehir Osmangazi University School of Medicine, Turkey, and meets the standards defined in the Helsinki Declaration.

Microscopic examination. For each of the sputum samples, we prepared two direct smear preparations and two homogenized decontaminated preparations. Decontamination and concentration processes were performed using the $\mathrm{N}$-acetyl-L-cysteine/ $\mathrm{NaOH}$ method (Decontamination and Concentration kit; RTA Laboratories), according to the manufacturer's instructions.

Kinyoun carbol fuchsin (cold carbol fuchsin) staining. For this staining, we prepared one direct sputum sample slide and one decontaminated sputum sample slide for each patient. The slides were stained with Kinyoun carbol fuchsin and 300 microscopic fields were examined under $\times 1000$ magnification.

Fluorochrome staining. We used the auramine phenol method for fluorochrome staining for which we prepared one direct sputum sample slide and one decontaminated sputum sample slide for each of the patients. The slides were stained with a 05151 Fluorescent Stain kit for Mycobacteria (Fluka) and examined under $\times 250$ (for at least 30 microscopic fields) or $\times 450$ (for at least 50 microscopic fields) magnification using fluorescence microscopy. The presence of yellow-green, thin, curled, granular bacteria on a dark background was interpreted as positive. The morphologies of bacteria showing fluorescence were further confirmed under $\times 1000$ magnification.

Culture. For culture studies, $0.1 \mathrm{ml}$ homogenized decontaminated sputum sample was inoculated onto solid slant LJ medium and incubated at $37{ }^{\circ} \mathrm{C}$ for up to 8 weeks. LJ medium slants were visually inspected every 2 days for mycobacterial growth. If growth was detected, slides were prepared from suspected colonies. The slides were then stained with carbol fuchsin and inspected to confirm the growth of AFB.

Before the inoculation, $0.1 \mathrm{ml}$ PANTA (Becton Dickinson), containing polymyxin, azlocillin, nalidixic acid, trimethoprim and amphotericin B, was added to BACTEC $12 \mathrm{~B}$ vials to prevent contamination. Next, $0.4-0.5 \mathrm{ml}$ homogenized decontaminated sputum sample was inoculated into the BACTEC $12 \mathrm{~B}$ vials, which were incubated at $37{ }^{\circ} \mathrm{C}$ and read twice a week for growth using a BACTEC 460 TB automated instrument. BACTEC 12B vials were read daily once the growth index (GI) was $\sim 10$. When the GI approached 100 , slides were prepared from the liquid medium, stained with carbol fuchsin and examined to confirm the growth of AFB. Liquid cultures were considered positive only when smears confirmed the presence of mycobacteria. If the GI was $<10$ at the end of 6 weeks, they were considered negative.

We also used a BACTEC NAP ( $p$-nitro- $\alpha$-acetylamino- $\beta$-hydroxypropiophenone) test (Becton Dickinson) to discriminate the MTBC from non-TB mycobacteria. The BACTEC NAP test selectively inhibits the growth of MTBC, but does not inhibit or only partially inhibits the growth of other mycobacterial strains. To distinguish whether a mycobacterium was a member of the MTBC, $0.1 \mathrm{ml}$ sample was taken from a BACTEC $12 \mathrm{~B}$ vial that was positive for AFB growth and inoculated into a BACTEC NAP vial. The GI was measured daily for 10 days. If the GI did not increase (i.e. the inoculated bacteria did not grow), the bacterium was considered to be MTBC.

We used the GTMD test for rapid identification of the following strains of mycobacteria: Mycobacterium avium, Mycobacterium intracellulare, Mycobacterium kansasii, Mycobacterium malmoense and MTBC. The test was performed according to the manufacturer's instructions and as described previously (Franco-Alvarez de Luna et al., 2006). We included a positive and negative control for each sample. Briefly, the procedure was completed in three steps. The first part consisted of heat lysis and sonication of cells, followed by $23 \mathrm{~S}$ rRNA isolation using a magnetic bead capture method. The second part of the procedure comprised isothermic amplification of RNA using a nucleic acid sequence-based amplification technique. Lastly, reverse hybridization of the amplified products was performed using an automated line probe assay system (Auto-LiPA; Innogenetics) (Boden et al., 1998). At the end of the test, the strips were interpreted using the interpretation chart provided by the manufacturer. The assay time was approximately $5-6 \mathrm{~h}$, allowing the results to be generated in 1 working day.

\section{RESULTS AND DISCUSSION}

Overall, the results of the present study showed that 84 of the 115 sputum samples were found to be positive for mycobacterial growth on BACTEC $12 \mathrm{~B}$ medium, 73 were positive by growth on LJ medium and 95 were positive by the GTMD test. Overall, 17 sputum samples were negative with all of the methods studied and 98 were positive by at least one of the methods. In addition, we detected AFB in 86 sputum samples using direct microscopy.

To enhance sensitivity, we prepared two direct and two homogenized decontaminated sputum sample smears 
for each patient. When we inspected the homogenized decontaminated sputum sample smears, we detected AFB in 86 and 66 samples using the fluorescent and carbol fuchsin staining procedures, respectively. As all of the samples positive for AFB by carbol fuchsin staining were also positive by fluorescent staining, we used the results of fluorescent staining of smears prepared from homogenized decontaminated sputum samples for evaluation of direct microscopic examination. Carbol fuchsin staining results indicated that, whilst 15 of the 66 samples were negative for AFB growth in direct sputum samples, all 66 smears prepared from homogenized decontaminated sputum samples were positive for AFB growth. Similarly, although the 86 smears prepared from homogenized decontaminated sputum samples were positive for AFB growth with fluorescent staining, 10 of these smears prepared from direct sputum samples were negative for the presence of AFB. Following examination of these different samples, we described the number of bacteria in smears prepared from homogenized and decontaminated sputum samples as ' + ' for a few bacteria and ' ++ ' for moderate numbers of bacteria. Thus, the present findings indicated that AFB growth may not be detected in direct smears that have only a few bacteria. Moreover, whilst there were only a few AFB $($ ' + ') in direct smears stained with carbol fuchsin and prepared from small amounts of sputum, staining was negative for AFB in smears prepared from homogenized decontaminated sputum. This observation implied that the preparation of direct smears from sputum samples often fails to detect the growth of AFB and may be a waste of time and work force. However, the preparation of direct smears may be useful for small amounts of sputum samples, as the homogenization and decontamination procedures dilute the sample and lead to the loss of AFB.

A comparison of the staining and culture methods is shown in Table 1. With fluorescent staining, we detected AFB in seven out of 31 sputum samples that did not show any growth. These seven sputum samples were obtained from patients who were under anti-TB treatment. When

Table 1. Test results for the 115 patients in this study

Culture was carried out using BACTEC 12B or LJ medium.

\begin{tabular}{|cccccc|}
\hline & \multicolumn{2}{c}{$\begin{array}{c}\text { Culture-positive } \\
(\boldsymbol{n}=\mathbf{8 4})\end{array}$} & & \multicolumn{2}{c|}{$\begin{array}{c}\text { Culture-negative } \\
(\boldsymbol{n}=\mathbf{3 1})\end{array}$} \\
\cline { 2 - 3 } \cline { 5 - 6 } & $\begin{array}{c}\text { GTMD- } \\
\text { positive }\end{array}$ & $\begin{array}{c}\text { GTMD- } \\
\text { negative }\end{array}$ & & $\begin{array}{c}\text { GTMD- } \\
\text { positive }\end{array}$ & $\begin{array}{c}\text { GTMD- } \\
\text { negative }\end{array}$ \\
\hline $\begin{array}{c}\text { Smear-positive } \\
(n=86)^{*}\end{array}$ & 78 & 1 & & 6 & 1 \\
$\begin{array}{c}\text { Smear-negative } \\
(n=29)^{*}\end{array}$ & 4 & 1 & & 7 & 17 \\
Total $(\boldsymbol{n}=\mathbf{1 1 5})$ & $\mathbf{8 2}$ & $\mathbf{2}$ & & $\mathbf{1 3}$ & $\mathbf{1 8}$ \\
\hline
\end{tabular}

${ }^{\star}$ Homogenized, decontaminated fluorescent staining. compared with culture, the sensitivity and specificity of fluorescent staining were 94 and $77 \%$, respectively.

A comparison of the GTMD test results and direct microscopy is also illustrated in Table 1 . There were two GTMD-negative samples that were positive for fluorescent staining. One of these samples was obtained from a patient under anti-TB treatment and the bacterial density for this sample was determined as ' + '; this sample did not show growth in culture. We concluded that the positivity in direct microscopic inspection of this sample could have been due to the presence of dead bacteria. Likewise, the bacterial density for the other sample was determined as ' + '. This sample was taken from a patient not receiving anti-TB treatment and showed growth in culture within 18 days (BACTEC 12B medium). As we observed an internal control amplification band on the GTMD test strip, the presence of an inhibitor in the sample was not considered and the result was interpreted as a false-negative for the GTMD test. When compared with direct microscopic inspection, the sensitivity, specificity and positive predictive value (PPV) and negative predictive value (NPV) of the GTMD test were established as 98, 62, 88 and $90 \%$, respectively.

Eighty-four and 73 sputum samples were found to be positive in BACTEC 12B vials and LJ medium, respectively. All growth patterns on LJ medium were concordant with those of MTBC (light-cream coloured, rough, breadcrumblike colonies). All AFB growing in BACTEC 12B medium were identified as MTBC by the NAP test. In addition, 82/ 84 culture-positive sputum samples were also verified as GTMD-positive (Table 1). The remaining two samples were culture-positive but GTMD-negative. One of these with no detectable band on the GTMD test strip was interpreted as negative following direct microscopic inspection. The negativity of this sample for the GTMD test may have been due to the presence of only a few bacteria. Similarly, the bacterial density for the other sample following direct microscopic inspection was determined as ' + '; this sample was taken from a patient receiving no anti-TB treatment. As we detected an internal control amplification band on GTMD test strip, the occurrence of an inhibitor in the sample was not considered and the result was interpreted as false-negative for the GTMD test due to presence of only a few bacteria. Moreover, 13 sputum samples showing no growth in the culture were positive for the GTMD test; 11 of these were obtained from patients under anti-TB treatment and with six of these we detected AFB following direct microscopic assessment. The other two of the 13 sputum samples (no growth in culture but positive for the GTMD test) were AFB-negative and were taken from patients not receiving anti-TB treatment at the time of sampling and diagnosed with different lung diseases. However, no medical history was taken regarding $\mathrm{TB}$ infection for these two patients. When compared with culture, the sensitivity, specificity, PPV and NPV of the GTMD test were 97, 58, 86 and $90 \%$, respectively. When the culture results and clinical findings of 
samples of $11 / 27$ patients under anti-TB treatment, they were determined using the GTMD test to contain MTBC $(40.7 \%)$. This situation prompted us to wonder whether the bacilli detected were viable or not. However, this may not be determined by culture because, using the culture method, a lack of growth does not exclude the presence of bacilli, as growth can only be seen when the sample contains a particular number of bacilli.

Similar to our present study, Seagar et al. (2008) compared the GTMD test with culture by studying 54 smear-positive respiratory samples and showed that the sensitivity and specificity of the GTMD test were 80.5 and $75 \%$, respectively. Franco-Alvarez de Luna et al. (2006) compared the GTMD test with the Cobas Amplicor MTB assay by studying 128 samples (respiratory and others) and showed that the sensitivity and specificity of the GTMD test were 92 and $100 \%$, respectively. Likewise, Weizenegger et al. (2004) compared the GTMD test with culture in 523 samples and reported that the sensitivity and specificity of the GTMD test were 82 and $99 \%$, respectively. Our results are in line with other studies, suggesting that the GTMD test can be used to detect TB infection with a high sensitivity ( $97 \%)$. The low specificity ( $58 \%$ ) of the GTMD test is a result of decreased growth of mycobacteria in sputum samples obtained from anti-TB-treated patients. When clinical findings and culture were assessed collectively, the specificity of the test increased to $90 \%$.

In conclusion, the results of the GTMD test can be obtained within 1 day and its strips are easy to interpret. Our observations suggest that the GTMD method can be useful for the early diagnosis of clinically and radiologically suspicious TB cases where smears are negative for mycobacteria. In addition, the use of the GTMD test for smear-positive cases is helpful and practical to differentiate MTBC and mycobacteria other than tuberculosis. This allows more rapid treatment decisions and infection-control precautions. The high cost of this test is the main obstacle to its use in the routine diagnosis of TB infection. Nevertheless, further studies with larger quantities of sputum samples and other clinical samples such as blood, faeces and urine are necessary. The efficacy of the GTMD test in determining living bacilli in TB patients under treatment should also be compared with more sensitive techniques.

\section{ACKNOWLEDGEMENTS}

This work was supported by the Research Projects Commission of Eskisehir Osmangazi University (project number: 2006/11023). Part of this manuscript was presented as a poster presentation at the 108th General Meeting of American Society for Microbiology in Boston in 2008.

\section{REFERENCES}

American Thoracic Society Workshop (1997). Rapid diagnostic test for tuberculosis. What is the appropriate use? Am J Respir Crit Care Med 155, 1804-1814.
Boden, D., Weizenegger, M., Benz, K., Ponstingl, W., Hengstler, M., Rusch-Gerdes, S., Fahr, A. \& Bartel, J. (1998). Reverse hybridization assay for rapid identification of mycobacteria from cultured samples. Clin Lab 44, 687-692.

Corbett, E. L., Watt, C. J., Walker, N., Maher, D., Williams, B. G., Raviglione, M. C. \& Dye, C. (2003). The growing burden of tuberculosis: global trends and interactions with the HIV epidemic. Arch Intern Med 163, 1009-1021.

Drobniewski, F. A., Caws, M., Gibson, A. \& Young, D. (2003). Modern laboratory diagnosis of tuberculosis. Lancet Infect Dis 3, 141-147.

Franco-Alvarez de Luna, F., Ruiz, P., Gutiérrez, J. \& Casal, M. (2006). Evaluation of the GenoType Mycobacteria Direct assay for detection of Mycobacterium tuberculosis complex and four atypical mycobacterial species in clinical samples. J Clin Microbiol 44, 3025-3027.

Hellyer, T. J., Fletcher, T. W., Bates, J. H., Stead, W. W., Templeton, G. C., Cave, M. D. \& Eisenach, K. D. (1996). Strand displacement amplification and the polymerase chain reaction for monitoring response to treatment in patients with pulmonary tuberculosis. J Infect Dis 173, 934-941.

Marks, G. L. (1993). Genetics of tuberculosis. Med Clin North Am 77, 1219-1233.

Metchock, B. G., Nolte, F. S. \& Wallace, R. J. (1999). Mycobacterium. In Manual of Clinical Microbiology, 7th edn, pp. 399-437. Edited by P. R. Murray, E. J. Baron, M. A. Pfaller, F. C. Tenover \& R. H. Yolken. Washington, DC: American Society for Microbiology.

Murray, P. R., Rosenthal, K. S. \& Pfaller, M. A. (2005). Mycobacterium. In Medical Microbiology, 5th edn, pp. 297-310. Philadelphia: Elsevier Mosby.

Piersimoni, C. \& Scarparo, C. (2003). Relevance of commercial amplification methods for direct detection of Mycobacterium tuberculosis complex in clinical samples. J Clin Microbiol 41, 5355-5365.

Piersimoni, C., Scarparo, C., Piccoli, P., Rigon, A., Ruggiero, G., Nista, D. \& Bornigia, S. (2002). Performance assessment of two commercial amplification assays for direct detection of Mycobacterium tuberculosis complex from respiratory and extrapulmonary specimens. J Clin Microbiol 40, 4138-4142.

Seagar, A.-L., Prendergast, C., Emmanuel, F. X., Rayner, A., Thomson, S. \& Laurenson, I. F. (2008). Evaluation of the GenoType Mycobacteria Direct assay for the simultaneous detection of the Mycobacterium tuberculosis complex and four atypical mycobacterial species in smear-positive respiratory specimens. J Med Microbiol 57, 605-611.

Singh, P., Kant, L., Shah, B., Muthuswamy, V. \& Saxena, N. C. (2002). What is new in the diagnosis of tuberculosis? Part I: Techniques for diagnosis of tuberculosis. Indian Counc Med Res Bull 32. http://www.icmr.nic.in/buaug02.pdf

Van der Vliet, G. M., Schepers, P., Schukkink, R. A., van Gemen, B. \& Klatser, P. R. (1994). Assessment of mycobacterial viability by RNA amplification. Antimicrob Agents Chemother 38, 1959-1965.

Weizenegger, M., Hengstler, M., Feldmann, K. \& Fahr, A. (2004). Direct detection of Mycobacterium tuberculosis-complex and four nontuberculosis mycobacteria: preliminary data of an evaluation in a hospital and a private laboratory [poster presentation]. In 25th Annual Congress of the European Society of Mycobacteriology (ESM), 27-30 June 2004, Alghero, Sardinia, Italy.

WHO (2007). Global tuberculosis control - surveillance, planning, financing. WHO/HTM/TB/2007.376. http://www.who.int/tb/ publications/global_report/2007/en.

Woods, G. L. (2001). Molecular techniques in mycobacterial detection. Arch Pathol Lab Med 125, 122-126. 